\title{
Application of Physical Factors in Complex Etiopathogenetic Therapy Patients with Coronavirus-19
}

\author{
Bitsoev Vladimir Dodtievich* \\ Academy of medical and technical Sciences, Russia
}

ISSN: 2637-7748

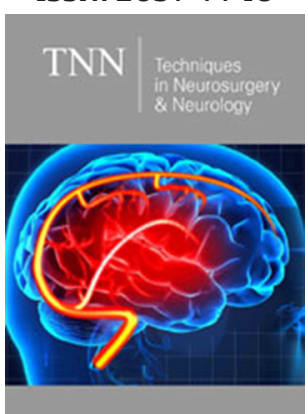

*Corresponding author: Bitsoev Vladimir Dodtievich, Academy of medical and technical Sciences, Russia

Submission: 海 July 01, 2020

Published: 侮June 20, 2020

Volume 3 - Issue 3

How to cite this article: Bitsoev Vladimir Dodtievich. Application of Physical Factors in Complex Etiopathogenetic Therapy Patients with Coronavirus-19. Tech Neurosurg Neurol. 3(3). TNN. 000564. 2020.

DOI: 10.31031/TNN.2020.03.000564

Copyright@ Bitsoev Vladimir Dodtievich, This article is distributed under the terms of the Creative Commons Attribution 4.0 International License, which permits unrestricted use and redistribution provided that the original author and source are credited.

\begin{abstract}
Fundamental scientific research of domestic and foreign scientists strongly suggests that the progress of medicine is impossible without the widespread use of modern physical factors in the diagnosis, prevention, treatment and rehabilitation of almost all nosologically forms of diseases from newborns to old age of patients. Each micro-and macro-organism has individual bioenergetic characteristics corresponding to its type, which is the main condition for normal life activity of the organism. In the case of "alien" bioenergetic characteristics, specific biological processes inherent in this organism are disrupted, which leads to its death [1]. The biopotential of each person is strictly individual in both normal and pathological conditions. The degree of deviation of the biopotential corresponds to the stage of development of the disease, i.e. the formation of intermediate States of the body with a violation of its supramolecular structures.
\end{abstract}

Keywords: Etiopathogenetic therapy; Supramolecular structures; Coronavirus; COVID-19

\section{Introduction}

Natural and preformed physical factors occupy an important place in medicine of the XXI century, as they are important for the diagnosis, treatment, prevention, and medical rehabilitation of patients.

\section{Medicine should be developed according to this concept}

"The level of medical excellence determines the progress of conservative treatment of patients with any pathology other than traumatology." Surgery should not be a factor in determining the progress of medicine. It is forced and will yield to the conservative method of treatment constantly with different speed and scale. Based on the progress of physical and technical Sciences, it is necessary to replace the concept of "mechanism of action of the physical factor" with "mechanism of interaction of the physical factor and the whole organism" [1-9]. Already from the moment of contact of the physical factor and the organism, intermediate States of both sides are formed with the release of energy (for the continuation of the cyclic process). In the Russian and world literature, we have not found scientific studies of their significance for the diagnosis, effectiveness and correction of treatment, prevention, and medical rehabilitation. This is especially important in Oncology for monitoring and managing the treatment process [3,5]. For the body, any external influence is a violation of its integrity and therefore the system of rapid disposal of it is instantly activated. Even your own blood outside the vascular bed is a foreign body for the body. That is, there is an instantaneous transfer of the work of all organs and systems of the body to an abnormal mode [4,5]. It should be noted that at the moment of development of medical science there are no methods for determining the time level of the cycle of each system at the time of exposure to the body of a medicinal substance, in its transition States at the supramolecular level and when all systems return to normal mode of life [10-15]. In this regard, it is difficult to imagine the advantage of a particular drug, and in particular for a particular patient. The lack of data in the chronological sequence of the drug in its path to achieving the goal calls into question the advantages of drug therapy over physical therapy.

It is known that " the interaction of a drug with the body is studied in two aspects: how it affects the body (pharmacodynamics) and what happens to it in the body (pharmacokinetics). 
Pharmacodynamicsstudies the localization, mechanismofactionand pharmacological effects of medicinal substances. Pharmacokinetics studies the patterns of absorption, distribution, and elimination of medicinal substances in the human and animal bodies [2]. At the same time, it should be noted that the speed, scale, content, and time of formation of intermediate formations of a pharmacological drug in the body for each patient are strictly individual. All drugs in interaction with the body before their introduction into medical practice, in accordance with pharmacodynamics, pharmacokinetics should be studied by fast, harmless, and highly informative methods [5]. Compliance with this concept contributes to ensuring a high therapeutic effect, primary and secondary prevention of diseases, prevention of complications and side effects on the body.

This is not observed in medical and pharmaceutical practice due to the lack of research methods for pharmacological preparations at the supramolecular level [6]. The biopotential for each person is strictly individual both in norm and in pathology. In this regard, any nosology in everyone causes a deviation of its biopotential in accordance with the stage of development of the disease, i.e. the formation of intermediate States of the body with certain violations of its supramolecular structures. This, in turn, determines the clinical picture at the time of examination of the patient and is the leading condition for choosing the right treatment tactics for any specialist doctor, so that the regression of the disease is accompanied by the restoration of destroyed supramolecular structures, excluding new gross violations at any level of the entire body [6,7]. This concept is not the main principle for drug therapy, due to the lack of highly informative research methods: frequency, dose, mechanism of action of pharmacological drugs at the supramolecular level. It is known that the final characteristic of any drug at the supramolecular level is "energy", which is difficult to dose and regulate for therapeutic purposes. The impact of energy from any physical factor is dosed and regulated (physio dynamics) using nanotechnology and its path can be freely traced to each molecule of the whole organism (physio kinetics) without disrupting supramolecular structures, without negative consequences by means of a Nano sensor.(patents for inventions: Russia \# 2675006, Germany \# 202017006 896.)

Modern pharmaceutical science does not have such a high level of control over the path of a medicinal substance in the body. "The conversion of the energy of photons, light particles, into electrical energy takes place in several stages," explains Professor Christoph Well, head of the IFG Institute. First, light is absorbed on the surface of the light-sensitive material [11]. Under the influence of the energy of photons of light, the electrons leave their places, leaving in their place electronic holes, with which they immediately form quasiparticles called polaritons. These polaritons exist only for a very short time, moving to the boundaries of the material, where they break up into electrons and holes, which continue to move further on their own. And the future fate of these charge carriers already depends on the nature of the light-sensitive material used" [11]. In this regard, any therapeutic effect on the body should be considered a trigger for restoring homeostasis, connecting its own internal systems. After studying the officially proposed and published Russian media medicines designed to combat coronavirus-19, we must admit not only their ineffectiveness, but sometimes even harm. Their original assumptions are clearly wrong. In fact, physiotherapy is seen as a more appropriate approach. It is based not only on the stated considerations, but also on half a century of experience in use.

\section{Highly effective methods of physical therapy include}

I. Light Therapy of the device "BIOPTRON"

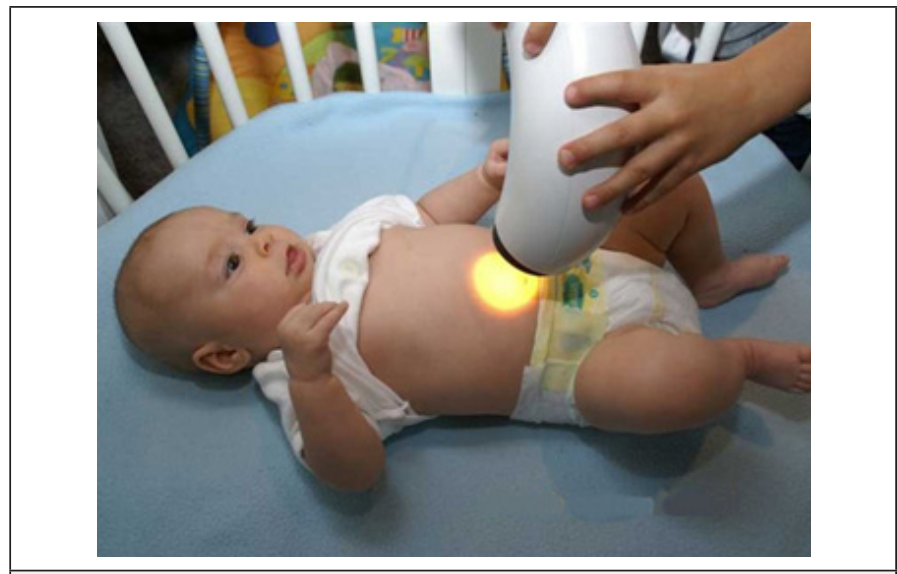

Figure 1: Light Therapy (PVIP) performed by the "BIOPTRON-COMPACT" device- $5 \mathrm{~cm}$.
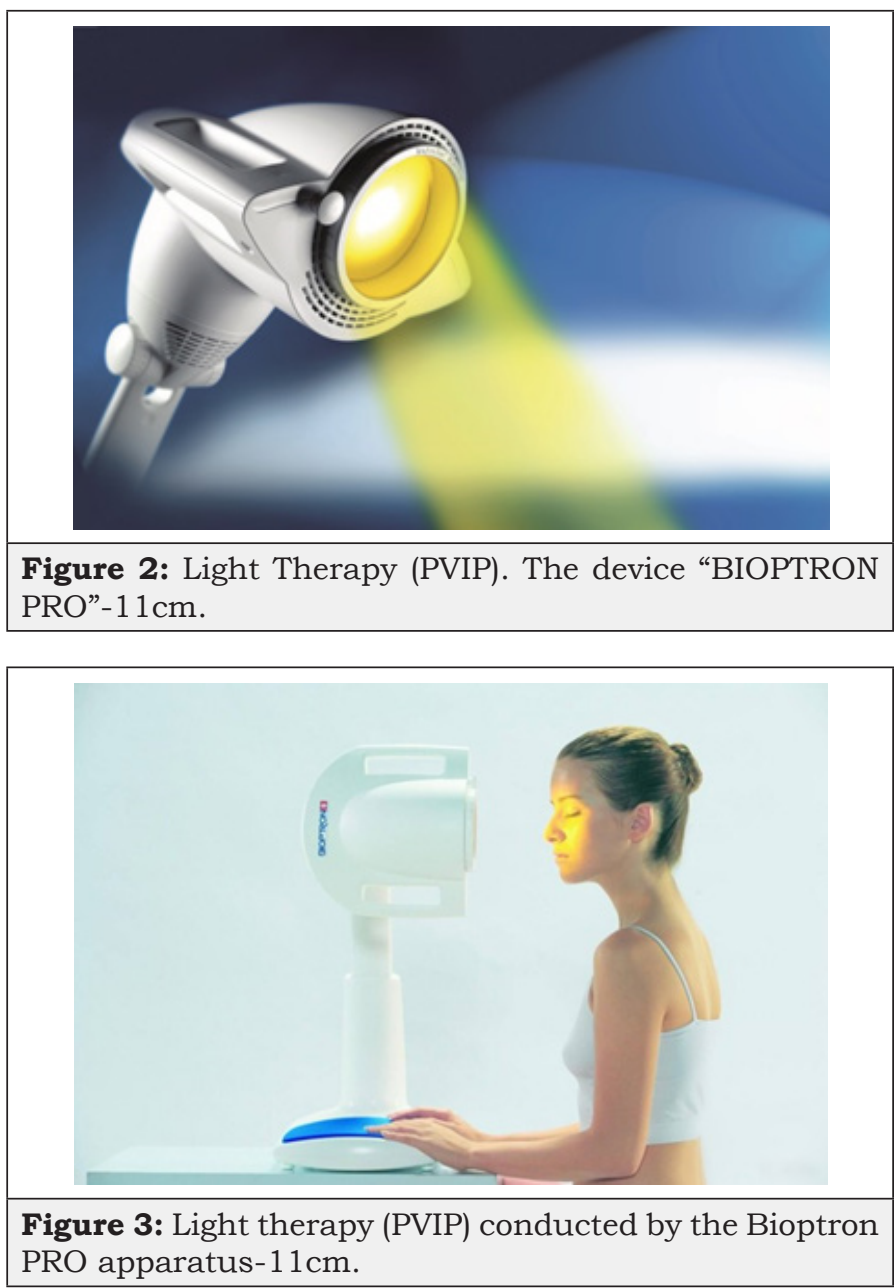
Its spectral range-480-3400 nm-reproduces the dominant types of Solar radiation on Earth-visible and IR radiation, under the influence of which the body absorbs and uses radiant energy. Polychromatic visible and infrared polarized (PVIP) light activates the enzymes nicotinamidadenin-dinucleotide phosphate-oxidase (NADP-oxidase) and nucleotide containing biopteroflavoproteinNO-synthesis, localized in the cell membrane and using the surrounding oxygen produce its active forms-superoxide anion, hydrogen peroxide, hydroxyl radical and nitric oxide (NO) [15]. They conduct a light signal from the surface of the irradiated cell to its nucleus, affecting specialized intracellular mechanisms for conducting the activation signal (protein phosphorylation, the state of calcium channels, the content of calcium in the cell, etc.). The enzymes responsible for the formation of ROS and NO, as themselves and intermediaries, are found in cells and tissues, in all types of white blood cells, platelets, endothelial and smooth muscle cells of blood vessels. It was found that nitric oxide-NO, is an important part of the mechanism of blood vessel dilation and platelet aggregation, without which phototherapy could hardly be highly effective $[10,14]$. After daily 5-10 irradiations, the number of mononuclear leukocytes-monocytes and lymphocytes-circulating in the blood increases by $14-17 \%$. 30 minutes after the first exposure to PVIP light, Pro-inflammatory cytokines-tumor necrosis factor (TNF- $\alpha$ ), interleukins-IL-6, IL-2, and IL-12-disappear from the circulating blood. So, at the initial increased content of TNF- $\alpha$, it falls by 30 times, IL-8-by 4-6 times, IL-2-by 4-10 times and IL12 -by 12 times, by the end of the course $[14,16]$. Simultaneously, the plasma content of anti-inflammatory cytokines-IL-10 and transforming growth factor-TFR- $\beta 1$ increases [14]. A feature of PVIP-light phototherapy is a rapid 6-fold increase in the blood of the most important immunomodulator-interferon- $\gamma$ (IFN- $\gamma$ ). The most important function of this cytokine is to activate cellular immunity (the functional state of monocytes, macrophages, natural killers, and cytotoxic T-lymphocytes), which primarily increases the body's antiviral and antitumor resistance [13] (Figures 1-3).

\section{Application of dry carbon dioxide baths "Reabox"}

Dry carbon dioxide baths (SUV) - a method of percutaneous therapeutic action of carbon dioxide on a patient whose body is located up to the neck level in a specially equipped box. Application (SUV) "Reabox" provides non-invasive, i.e. does not violate the integrity of the skin, the introduction of carbon dioxide, which distinguishes this method from $\mathrm{CO} 2$ injections. Direct action of carbon dioxide on the respiratory center. The excitation of the respiratory center is not caused by carbonic acid itself, but by an increase in the concentration of hydrogen ions due to an increase in its content in the cells of the respiratory center. The specificity of carbonic acid as a respiratory center pathogen was revealed by the experiments of Frederick and Holden, who found that $\mathrm{h}+$ and HCO3 ions pass poorly through the cell membrane, and undifferentiated carbonic acid passes well: undifferentiated $\mathrm{H} 2 \mathrm{CO} 3$ diffuses into the cells of the nerve center, which dissociates already in the nerve cells, releasing the irritating $\mathrm{H}+$ ion. Faster diffusion into cells than other acids is a specific feature of carbonic acid, and this is associated with a stronger irritating effect on the respiratory center $[12,15]$.
Hyperventilation for a short time (several tens of minutes) leads to death due to the loss of carbon dioxide by the body. Humoral regulation of respiration, the role of carbon dioxide, oxygen, and blood $\mathrm{pH}$ in this process. The main respiratory stimulant is $\mathrm{CO}_{2}$. Blood $\mathrm{pH}$ also plays an important role in the regulation of respiration. When the $\mathrm{pH}$ of arterial blood decreases in comparison with the normal level (7.4), lung ventilation increases, and when the $\mathrm{pH}$ increases above the norm, ventilation decreases. Increasing the content of $\mathrm{CO} 2$ in the blood stimulates respiration both by reducing the $\mathrm{pH}$ and directly by the action of $\mathrm{CO}_{2}$ itself $[12,15]$. The effect of $\mathrm{CO} 2$ and $\mathrm{H}+$ ions on respiration is mediated mainly by their action on special structures of the brain stem that have chemosensitivity (Central chemoreceptors are part of the blood-brain barrier; low sensitivity threshold). It was found that a decrease in the $\mathrm{pH}$ of the cerebrospinal fluid by only 0.01 is accompanied by an increase in pulmonary ventilation by $4 \mathrm{l} / \mathrm{min}$. [15]

Lack of $\mathrm{O}_{2}$ can be a respiratory stimulant in the case of barbiturates as narcotic drugs, because in this case, the sensitivity of the respiratory center to $\mathrm{CO}_{2}$ is suppressed. Breathing pure oxygen $\left(\mathrm{O}_{2}\right)$ in patients with reduced sensitivity to $\mathrm{CO}_{2}$ is very dangerous, because when the $\mathrm{O}_{2}$ voltage increases in the arterial blood the last respiratory stimulant (lack of $\mathrm{O}_{2}$ ) is eliminated in the blood and respiratory arrest may occur. In such cases, it is necessary to use an artificial respiration device (Table 1).

Table 1: The normal ranges of total $\mathrm{CO}_{2}$ in the blood should be as follows.

\begin{tabular}{|c|c|c|}
\hline Age Range & Conventional Units & SI units \\
\hline $18-59$ & $23-29 \mathrm{mEq} / \mathrm{l}$ & $23-29 \mathrm{mmol} / \mathrm{l}$ \\
\hline $60-89$ & $23-31 \mathrm{mEq} / \mathrm{l}$ & $23-31 \mathrm{mmol} / \mathrm{l}$ \\
\hline $90+$ & $20-29 \mathrm{mEq} / \mathrm{l}$ & $20-29 \mathrm{mmol} / \mathrm{l}$ \\
\hline
\end{tabular}

III. Extremely high frequency therapy (EHF) is the therapeutic use of millimeter-wave electromagnetic waves

The experience of using it for more than 30 years shows high efficiency in the treatment of a wide range of diseases, including cancer patients. Extremely high frequencies occupy the range of $30-300 \mathrm{GHz}$ (the wavelength range is $10-1 \mathrm{~mm}$ ). The peculiarity of this frequency range is that millimeter radiation of cosmic origin is almost absorbed by the earth's atmosphere, so the biological evolution of all living organisms took place with a very small natural EHF electromagnetic background. This, apparently, explains the active influence of low-intensity millimeter radiation on a person. The following wavelengths are most used in EHF therapy: $4.9 \mathrm{~mm}$ (60.12 GHz), $5.6 \mathrm{~mm}$ (53.33 GHz), and $7.1 \mathrm{~mm}(42.19 \mathrm{GHz}$ ) [8]. Lowintensity millimeter radiation refers to non-ionizing radiation, i.e. it cannot have a destructive harmful effect on the biological tissues of the body, and therefore it is safe. A specific feature of EHF exposure is its normalizing nature, i.e. EHF radiation normalizes only the physiological parameters of a number of States of the body that deviate from it: it increases the values of reduced indicators and reduces the values of inflated values. Parameters that are normal do not respond to radiation of the body with a millimeter field. That is, the features of EHF therapy as non-invasiveness, lack of Allergy 
to EHF radiation, drug-free therapy contribute to the normalization of intracellular energy of any cell in the whole body (Figures 4-6).

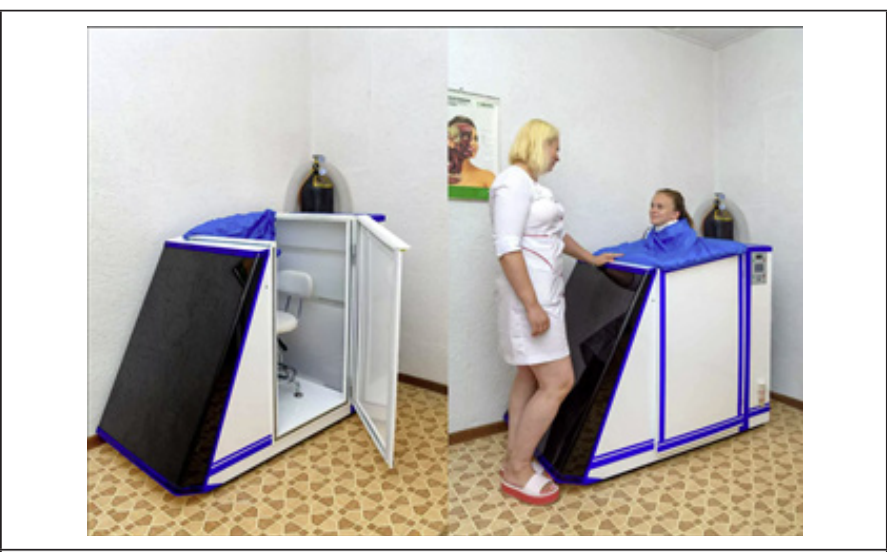

Figure 4: Dry carbon dioxide baths using the "Reabox" device.

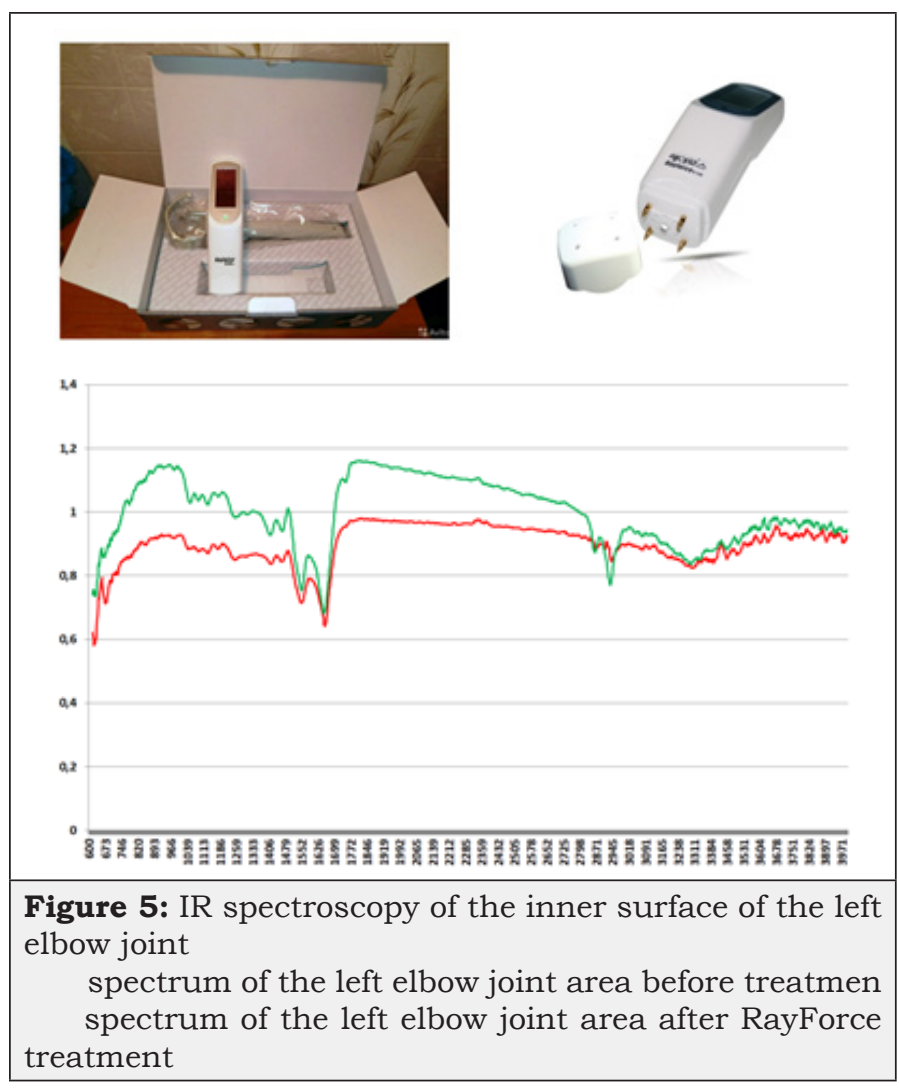

IV. Multifunctional device for spot infrared and magnetic therapy for effective pain relief (Rayforce)

IR wavelength: $850 \mathrm{~nm}$. Magnet power: 1000 Gauss. Charging: from sunlight and artificial light. IR-therapy. It is proved that waves of different ranges affect the body in different layers and levels. IR radiation has the greatest penetration depth. In physiotherapy, waves are used in the range from 780 to $1400 \mathrm{~nm}$, i.e. short, penetrating the tissues to a depth of $5 \mathrm{~cm}$. The effect of IR radiation is aimed at accelerating the physical and chemical processes reactions: the processes of tissue repair and regeneration are stimulated, the vascular network expands, blood flow accelerates, cell growth increases, biologically active substances are produced, and white blood cells are sent to the lesion site. The reserve functions of the body are awakened. Permanent magnetic field (PMP) improves microcirculation, stimulates healing processes, activates immunological reactions, has anti-inflammatory and sedative effects $[16,17]$. Experimental studies were conducted at the scientific center for fiber optics (ncvo) of the Russian Academy of Sciences, Moscow. New non-toxic, non-hygroscopic silver halide light guides with low optical losses in a wide spectral range of 3-15 microns have been developed by the ntsvo staff, allowing to obtain skin spectra in vivo with a good signal-to-noise ratio even on the uncooled standard DNGS Fourier spectrometer Bruker. Results of RayForce treatment effectiveness based on IR spectroscopy data. This experiment confirmed the high therapeutic effectiveness of the RayForce device: For rice.5 IR spectroscopy shows the absence of pain in the left elbow joint after the treatment of RayForce device in the form of a complete restoration of the spectrum in the form and amplitude of light transmission, as well as correction of morphological changes in this area of exposure in the wavelength range of $970-1400 \mathrm{~nm}$. Based on the data in Fig.6. according to IR spectroscopy, there is reason to assert that the right elbow joint in the experiment was healthy and should be considered IR spectroscopy of the right elbow joint control.

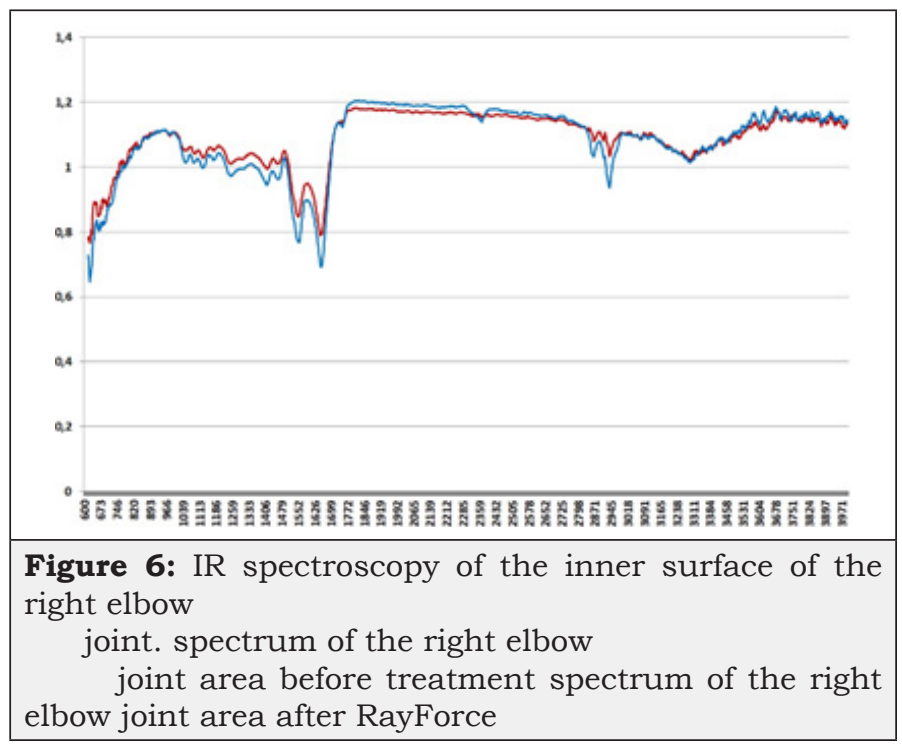

Symptoms of coronavirus, the first signs of COVID-19.

a. Elevated temperature

b. Dry cough

c. Dyspnea

d. The tightness in his chest

e. Runny nose

f. Weakness

g. Undue fatigability

The lung involvement by coronavirus the structure of human lungs is cellular. They consist of tiny bubbles saturated with air - 
the alveoli. Each such alveola is surrounded by capillaries, through which, in fact, carbon dioxide is removed from the blood and oxygen is supplied. Red blood cells (red blood cells) are responsible for their transport through tissues and organs. Alveolar cells that participate in gas exchange are of two types: type I. Thin. Oxygen passes through them; Type II. A surfactant is isolated - a substance that envelops the alveola and protects it from damage. The coronavirus attacks mainly type II cells. Spiked proteins on its surface are bound by angiotensin converting enzyme 2 (APF2) on their surface. So, the virus "breaks" the protection and gets inside the cell, starting to replicate its RNA. The host cell soon dies, and the coronavirus spreads to neighboring cells and thus gradually affects the lungs. Naturally, our immune system does not sit still and actively produces macrophage cells. The result of this struggle is the death of the alveoli and a decrease in the turnover of gas exchange. This continues until the so-called alveolar collapse occurs and the acute respiratory distress syndrome begins. In severe inflammation, fluid rich in inflammatory proteins enters the bloodstream and spreads to other organs and tissues. This is how the systemic inflammatory response syndrome (SARS) develops, followed by septic shock and multiple organ failure. The incubation period of COVID-19 is from 2 to 14 days, during which time there are no symptoms. Preventive physical therapy should be started as soon as a coronavirus infection is suspected.

a. light Therapy with the "BIOPTRON" device

b. Impact on the face from $10 \mathrm{~cm}$ to 5 minutes

c. On the neck area with $10 \mathrm{~cm} 5$ minutes

d. On the inter-scapular area with $5 \mathrm{~cm} 10$ minutes

e. $\quad$ n the plantar surfaces of the feet from $5 \mathrm{~cm}$ to 5 minutes

f. On Palmar surfaces from $5 \mathrm{~cm}$ to 5 minutes

g. Twice a day, daily, the course of treatment is 14 days

h. Dry carbon dioxide baths "Reabox"

i. $\quad \mathrm{CO}_{2}$ concentration of $18-20 \%, 15$ minutes, 1 time per day, treatment course of 14 days

j. EHF therapy, the device "Yav"

k. Paravertebral inter-scapular area with two points on both sides, the distance between the points is $10 \mathrm{~cm}$

\section{l. Epigastric region}

m. Paravertebral at the level of the VII cervical vertebra

n. $\quad$ Emitters: $4.9 \mathrm{~mm}(60.12 \mathrm{GHz}), 5.6 \mathrm{~mm}(53.33 \mathrm{GHz})$ and $7.1 \mathrm{~mm}(42.19 \mathrm{GHz})$ for 3 minutes per field 1 time per day, daily, 14 days of treatment

Therapeutic breathing exercises 5 minutes, 1 time a day, daily, 14 days of treatment. In case of complications, the physiotherapist plans of daily physiotherapy with hourly correction individually for each patient according to the state of the clinic. Physical therapy is performed in combination with medication. The proposed physiotherapy plan provides indications and contraindications at the supramolecular level, as well as for children from two years of age with a $50 \%$ reduction in the exposure time of each method, i.e. if an adult is 5 minutes, then children are 2.5-3 minutes. When establishing the diagnosis of "pneumonitis", the oxygen-helium mixture should be inhaled according to the developed method of academician A. G. Chuchalin from the AKGS-31 apparatus of the Minsk research Institute of radio materials.

\section{Conclusion}

Given the characteristics of coronavirus (COVID-19) infection, its differences from other known viruses are

1) suddenness of occurrence

2) high speed, scale and unhindered distribution

3) program selectivity of penetration into the intracellular space

4) Consistency of the striking nature at the supramolecular level of chronically weakened organs and systems, considering their biological age.

The wave origin of coronavirus-19, that is, based on quantum mechanics (entanglement), should be assumed. In this regard, it should be argued that a global solution to the problem of neutralizing the damaging insidious actions of the virus (COVID-19) is possible at the level of quantum physics and can only be done by a group of scientific physicists led by professor Lukin Mikhail Dmitrievich of the United States Harvard University. The physiotherapeutic methods proposed above for the prevention, treatment, and rehabilitation of patients with coronavirus (COVID-19) infection are also consistent with quantum physics, since their mechanism of action on the whole organism is identical to quantum touch, so they should be included in the program for combating coronavirus infection (COVID19).

\section{References}

1. Aldersons AA (1985) Mechanism of electrodermal reactions. USA.

2. Bertram GK (1998) Translation from english edited by Doctor of Medical Sciences, prof. Basic and clinical pharmacology 1(2).

3. Bitsoev VD (2012) System of restorative treatment of degenerative dystrophic lesions of the spine: abstract of Doctor of Medical Sciences. Russia.

4. Bitsoev VD (2015) New direction in the study of the role of interaction of the body and physical factors in complex therapy of patients. Russia.

5. Bitsoev VD (2015) The Application of corrective underwater spinal traction with underwater phototherapy to the spine pathology. Clinical Medicine Research 6(4): 204-213.

6. Bitsoev VD (2016) New approach in investigating the role of interaction between an organism and physical factors in complex patient therapy. Russia.

7. Garyaev P (1995) Wave genetic code. USA.

8. Devyatkov ND, Golant MB, Betsky OV (1987) Instructions for the use of a therapeutic installation for the treatment of millimeter-wave radiation of non-thermal intensity. USA.

9. Jean ML (1998) Supramolecular chemistry. Nauka. 
10. Malkoch AV, Maydannik VG, Kurbanova EG (2000) The Physiological role of nitric oxide in the body (Part 1).

11. Oldenburg Michael (2001) Photon up-conversion at crystalline organic-organic heterojunctions. Advanced Materials pp. 65.

12. Ryabov GA, Azizov YM (2002) The Role of nitric oxide as a regulator of cellular processes in the formation of multiple organ failure. Educational and scientific center of the MC UD of the President of the Russian Federation, Moscow.
13. Savinov VA, Samokhvalov EG (1997) Human energy System. Moscow.

14. Samoylova KA (203) The Mechanism of anti-inflammatory, immunomodulating, wound healing and normalizing metabolism of the light of the device "BIOPTRON", Moscow.

15. Sosunov AA (2000) Nitric Oxide as an intercellular mediator". Saransk. 16. Ulashchik VS (1994) Essays on general physiotherapy. pp. 198.

17. https://www.orbitlonglife.ru/zhizn-bez-boli/orbit-rayforce/

For possible submissions Click below: 